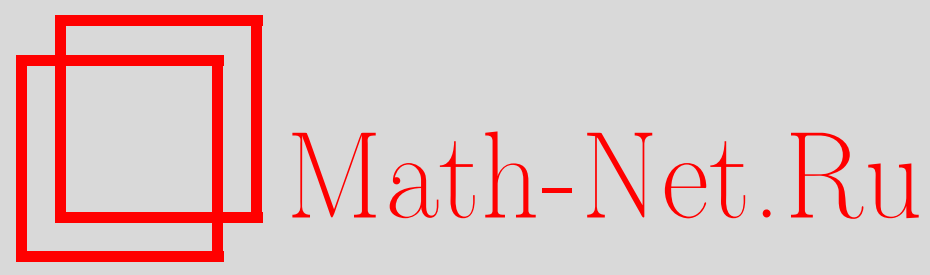

В. А. Краснов, Об ориентируемых вещественных алгебраических М-поверхностях, Матем. заметки, 1997, том 62, выпуск 4, 520-526

DOI: https://doi.org/10.4213/mzm1635

Использование Общероссийского математического портала Math-Net.Ru подразумевает, что вы прочитали и согласны с пользовательским соглашением http://www . mathnet.ru/rus/agreement

Параметры загрузки:

IP : 3.85 .73 .92

26 апреля 2023 г., 15:37:55

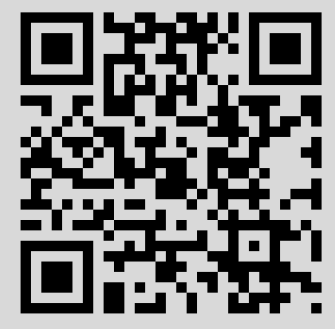




\title{
ОБ ОРИЕНТИРУЕМЫХ ВЕЩЕСТВЕННЫХ АЛГЕБРАИЧЕСКИХ $M$-ПОВЕРХНОСТЯХ
}

\author{
В. А. Краснов
}

\begin{abstract}
Изучаются соотношения между классами гомологий, определенных вещественными точками вещественной алгебраической $M$-поверхности. Доказываются новые сравнения для эйлеровых характеристик компонент множества вещественных точек такой поверхности.

Библиограффия: 9 названий.
\end{abstract}

Формулировка результатов. Пусть $X$ - неособая проективная вешественная алгебраическая $M$-поверхность, предполагается, что множество вешественных точек $X(\mathbb{R})$ ориентируемое, а группа гомологий $H_{*}(X(\mathbb{C}), \mathbb{Z})$ свободная. Пусть $X_{1}, \ldots, X_{s}-$ компоненты связности множества $X(\mathbb{R})$, они определяют классы гомологий $x_{1}, \ldots, x_{s} \in$ $H_{2}\left(X(\mathbb{C}), \mathbb{F}_{2}\right)$.

Теорема 1. Между классами гомологий $x_{1}, \ldots, x_{s}$ существует $q+1$ линейно независимых соотношений, где $q$ - иррегулярность поверхности $X$.

Заметим, что больше чем $q+1$ линейно независимых соотношений между классами гомологий $x_{1}, \ldots, x_{s}$ быть не может, этот факт доказан в [1]. Далее, через $\chi_{k}$ будем обозначать эйлерову характеристику компоненты $X_{k}, k=1, \ldots, s$.

Теорема 2. Eсли $\chi_{k} \equiv 0\left(\bmod 2^{\mu}\right), \mu \geqslant 1, k=1, \ldots, s, x_{i_{1}}+\cdots+x_{i_{n}}=0$, mo имеет место сравнение $\chi_{i_{1}}+\cdots+\chi_{i_{n}} \equiv 0\left(\bmod 2^{\mu+2}\right)$.

Если $H_{1}(X(\mathbb{C}), \mathbb{Z})=0$, то единственное соотношение между классами гомологий $x_{1}, \ldots, x_{s}$ имеет вид $x_{1}+\cdots+x_{s}=0$, для описания возможных соотношений в общем случае нужно применять отображение Альбанезе $\alpha: X \rightarrow A$. Множество комплексных точек многообразия Альбанезе $A(\mathbb{C})$ является комплексным тором с антиголоморфной инволюцией $\theta: A(\mathbb{C}) \rightarrow A(\mathbb{C})$. Так как $X-M$-поверхность, то $A$ является $M$-многообразием, и пару $(A(\mathbb{C}), \theta)$ топологически можно отождествлять с комплексным тором $\mathbb{C}^{q} / \mathbb{Z}^{q}$, на котором инволюция задается комплексньм сопряжением (см. [2], $[3])$. Множество вещественных точек $A(\mathbb{R})$ представляет собой объединение $2^{q}$ вещественных торов. Через $A(\mathbb{R})^{(i)}, i=1, \ldots, q$, обозначим объединение тех компонент $A(\mathbb{R})$, которые при проекции $\pi_{i}: \mathbb{C}^{q} / \mathbb{Z}^{q} \rightarrow \mathbb{C} / \mathbb{Z}, \pi_{i}\left(z_{1}, \ldots, z_{q}\right)=z_{i}$, отображаются в кривую $\mathbb{R} / \mathbb{Z} \subset \mathbb{C} / \mathbb{Z}$. Через $X(\mathbb{R})^{(i)}$ обозначим объединение компонент $X(\mathbb{R})$, которые при отображении Альбанезе $\alpha: X \rightarrow A$ отображаются в $A(\mathbb{R})^{(i)}$. 
Теорема 3. Классы гомологий $[X(\mathbb{R})],\left[X(\mathbb{R})^{(i)}\right], i=1, \ldots, q$, равны 0 в группе $\mathrm{H}_{2}\left(X(\mathbb{C}), \mathbb{F}_{2}\right)$, причем соответствующие им соотношения между классами гомологий $x_{1}, \ldots, x_{s}$ образуют базис соотношений.

В случае, когда базис отображения Альбанезе $\alpha: X \rightarrow A$ является кривой, появляется еще одна возможность получения базиса соотношений между классами гомологий $x_{1}, \ldots, x_{s}$. Итак, пусть $C=\alpha(X)-$ кривая, тогда $C$ не имеет особых точек (см. [4, c. 49]). Кривая $C$ будет $M$-кривой, так как гомоморфизм

$$
\alpha_{*}: H_{1}\left(X(\mathbb{C}), \mathbb{F}_{2}\right) \rightarrow H_{1}\left(C(\mathbb{C}), \mathbb{F}_{2}\right)
$$

является изоморфизмом, и инволюция комплексного сопряжения действует на группе $H_{1}\left(X(\mathbb{C}), \mathbb{F}_{2}\right)$ тривиально. Следовательно, число компонент связности множества $C(\mathbb{R})$ равно $q+1$, обозначим их через $C_{1}, \ldots, C_{q+1}$, а через $X(\mathbb{R})_{i}$ обозначим объединение компонент $X(\mathbb{R})$, которые отображаются в $C_{i}, i=1, \ldots, q+1$.

Теорема 4. Классы гомологий $\left[X(\mathbb{R})_{i}\right], i=1, \ldots, q+1$, равны 0 в $H_{2}\left(X(\mathbb{C}), \mathbb{F}_{2}\right)$, причем соответствующие им соотношения между классами гомологий $x_{1}, \ldots, x_{s}$ образуют базис соотношений.

Доказательство результатов. Сначала введем следующие обозначения. Пусть $G$ - группа Галуа $G(\mathbb{C} / \mathbb{R})$, она имеет второй порядок и действует на множестве комплексных точек $X(\mathbb{C})$. Факторпространство $X(\mathbb{C}) / G$ обозначим через $Y$, а проекцию $X(\mathbb{C}) \rightarrow Y$ - через $f$. Множество вешественных точек $X(\mathbb{R})$ можно рассматривать вложенным в $Y$, поэтому компоненты $X_{1}, \ldots, X_{s}$ определяют классы гомологий $y_{1}, \ldots, y_{s} \in H_{2}\left(Y, \mathbb{F}_{2}\right)$. Число линейно независимых соотношений между классами гомологий $y_{1}, \ldots, y_{s}$ можно вычислить следующим образом. Так как $Y$ является дифференцируемьм многообразием, то определен гомоморфизм Гизина

$$
j^{*}: H^{0}\left(X(\mathbb{R}), \mathbb{F}_{2}\right) \rightarrow H^{2}\left(Y, \mathbb{F}_{2}\right) .
$$

Обозначим через $\varkappa(Y)$ размерность ядра этого гомоморфизма, тогда число $\varkappa(Y)$ равно числу линейно независимых соотношений между классами гомологий $y_{1}, \ldots, y_{s}$. Итак, нужно вычислить число $\varkappa(Y)$. Гомоморфизм Гизина (1) включается в следующую точную последовательность (см. [5]):

$$
0 \rightarrow H^{1}\left(Y, \mathbb{F}_{2}\right) \rightarrow H^{1}\left(X(\mathbb{C}) ; G, \mathbb{F}_{2}\right) \rightarrow H^{0}\left(X(\mathbb{R}), \mathbb{F}_{2}\right) \stackrel{j^{*}}{\rightarrow} H^{2}\left(Y, \mathbb{F}_{2}\right),
$$

которая получается из первой спектральной последовательности эквивариантных когомологий $H^{*}\left(X(\mathbb{C}) ; G, \mathbb{F}_{2}\right)$. Следовательно, вьполняется равенство

$$
\varkappa(Y)=\operatorname{dim} H^{1}\left(X(\mathbb{C}) ; G, \mathbb{F}_{2}\right)-\operatorname{dim} H^{1}\left(Y, \mathbb{F}_{2}\right) .
$$

Так как $X$ является $M$-поверхностью, то

$$
\operatorname{dim} H^{1}\left(X(\mathbb{C}) ; G, \mathbb{F}_{2}\right)=1+\operatorname{dim} H^{1}\left(X(\mathbb{C}), \mathbb{F}_{2}\right)=1+2 q
$$

Покажем теперь, что

$$
\operatorname{dim} H^{1}\left(Y, \mathbb{F}_{2}\right)=q .
$$


Действительно, из того, что $X-M$-поверхность, а группа $H^{*}(X(\mathbb{C}), \mathbb{Z})$ свободная, следует, что $X$ является $G M \mathbb{Z}$-поверхностью (см. [6]), поэтому гомоморфизм

$$
f^{*}: H^{*}(Y, \mathbb{Z}) \rightarrow H^{*}(X(\mathbb{C}), \mathbb{Z})
$$

является вложением (см. [7]). Следовательно, группа $H^{*}(Y, \mathbb{Z})$ свободная, тогда

$$
\operatorname{dim} H^{1}\left(Y, \mathbb{F}_{2}\right)=\operatorname{dim} H^{1}(Y, \mathbb{Q})=\operatorname{dim} H^{1}(X(\mathbb{C}), \mathbb{Q})^{G}=q .
$$

Теперь из (3)-(5) получаем равенство $\varkappa(Y)=q+1$. Тем самьм, доказана

ЛЕмма 1. Число линейно независимых соотношений между классами гомолоәий $y_{1}, \ldots, y_{s}$ равно $q+1$.

Введем дополнительные обозначения. Выберем ориентацию на топологических поверхностях $X_{1}, \ldots, X_{s}$, тогда они определят классы гомологий в $H_{2}(X(\mathbb{C}), \mathbb{Z})$ и $H_{2}(Y, \mathbb{Z})$, которые обозначим через $\boldsymbol{x}_{1}, \ldots, \boldsymbol{x}_{s}$ и $\boldsymbol{y}_{1}, \ldots, \boldsymbol{y}_{s}$ соответственно. Гомоморфизм Гизина

$$
H_{2}(Y, \mathbb{Z}) \rightarrow H_{2}(X(\mathbb{C}), \mathbb{Z})
$$

для отображения $f: X(\mathbb{C}) \rightarrow Y$ обозначим через $f^{*}$, тогда вьполняются равенства

$$
\begin{aligned}
f^{*}\left(\boldsymbol{y}_{k}\right) & =2 \boldsymbol{x}_{k}, \quad k=1, \ldots, s, \\
H_{2}(X(\mathbb{C}), \mathbb{Z})^{G} & =f^{*} H_{2}(Y, \mathbb{Z})+\mathscr{L}\left(\boldsymbol{x}_{1}, \ldots, \boldsymbol{x}_{s}\right),
\end{aligned}
$$

где $\mathscr{L}\left(\boldsymbol{x}_{1}, \ldots, \boldsymbol{x}_{s}\right)$ - линейная оболочка элементов $\boldsymbol{x}_{1}, \ldots, \boldsymbol{x}_{s}$. Равенство (6) всегда выполняется, а равенство (7) следует из того, что $X$ является $G M \mathbb{Z}$-поверхностью (см. [5]). Покажем теперь, что справедлива

ЛЕмма 2. Из соотношения $\boldsymbol{y}_{i_{1}}+\cdots+\boldsymbol{y}_{i_{n}} \equiv 0(\bmod 2)$ следует соотношение $\boldsymbol{x}_{i_{1}}+\cdots+\boldsymbol{x}_{i_{n}} \equiv 0(\bmod 2)$.

ДокАЗАтЕльство. Действительно, так как $X-M$-поверхность, то имеет место разложение (см. [8])

$$
H_{2}(X(\mathbb{C}), \mathbb{Z})=H_{2}(X(\mathbb{C}), \mathbb{Z})_{+} \oplus H_{2}(X(\mathbb{C}), \mathbb{Z})_{-},
$$

где

$$
H_{2}(X(\mathbb{C}), \mathbb{Z})_{ \pm}=\left\{a \in H_{2}(X(\mathbb{C}), \mathbb{Z}) \mid \tau^{*}(a)= \pm a\right\}
$$

а $\tau: X(\mathbb{C}) \rightarrow X(\mathbb{C})$ - инволюция комплексного сопряжения.

Группы $H_{2}(X(\mathbb{C}), \mathbb{Z})_{+}, H_{2}(X(\mathbb{C}), \mathbb{Z})_{-}$ортогональны относительно индекса пересечения, $\boldsymbol{x}_{i_{1}}+\cdots+\boldsymbol{x}_{i_{n}} \in H_{2}(X(\mathbb{C}), \mathbb{Z})_{+}$, поэтому достаточно проверить, что индекс пересечения $a \cdot\left(\boldsymbol{x}_{i_{1}}+\cdots+\boldsymbol{x}_{i_{n}}\right)$ делится на 2 при каждом $a \in H_{2}(X(\mathbb{C}), \mathbb{Z})_{+}$. Но из $(6)$ получаем равенство

$$
\boldsymbol{x}_{i_{1}}+\cdots+\boldsymbol{x}_{i_{n}}=f^{*}\left(\frac{1}{2}\left(\boldsymbol{y}_{i_{1}}+\cdots+\boldsymbol{y}_{i_{n}}\right)\right)
$$

Осталось применить формулу (7) и равенства $x_{k}^{2}=-\chi_{k}, k=1, \ldots, s$. Лемма 2 доказана.

Заметим теперь, что из лемм 1, 2 следует теорема 1. 
ДоКАЗАТЕЛЬСТвО ТЕОРЕМЫ 2. Покажем сначала, что существует соотношение

$$
l_{1} \boldsymbol{x}_{1}+\cdots+l_{s} \boldsymbol{x}_{s} \equiv 0\left(\bmod 2^{\mu}\right)
$$

где числа $l_{i_{1}}, \ldots, l_{i_{n}}$ нечетные, а остальные числа $l_{k}$ четные. При $\mu=1$ существование соотношения (9) очевидно, так как $x_{i_{1}}+\cdots+x_{i_{n}}=0$. Пусть $\mu \geqslant 2$. Предположим, что соотношения вида (9) не существует, тогда обозначим через $\nu$ максимальное целое число, для которого существует соотношение

$$
l_{1} \boldsymbol{x}_{1}+\cdots+l_{s} \boldsymbol{x}_{s} \equiv 0\left(\bmod 2^{\nu}\right)
$$

где числа $l_{i_{1}}, \ldots, l_{i_{n}}$ нечетные, а остальные числа $l_{k}$ четные. Положим

$$
h=\frac{1}{2^{\nu}}\left(l_{1} \boldsymbol{x}_{1}+\cdots+l_{s} \boldsymbol{x}_{s}\right)
$$

и заметим, что можно так изменить числа $l_{1}, \ldots, l_{s}$, что $h \in f^{*}(Y, \mathbb{Z})$. Действительно, в силу разложения (7) существуют целые числа $m_{1}, \ldots, m_{s}$ такие, что

$$
h+\sum_{k=1}^{s} m_{k} \boldsymbol{x}_{k} \in f^{*} H_{2}(Y, \mathbb{Z}) .
$$

Осталось заменить $l_{k}$ на $l_{k}+m_{k} \cdot 2^{\nu}$. Так как $x_{k}^{2}=-\chi_{k}, \nu<\mu$, то в силу разложения (7) получаем, что индекс пересечения $a \cdot h$ является четным для любого элемента $a \in H_{2}(X(\mathbb{C}), \mathbb{Z})^{G}$, а в силу разложения $(8)$ он будет четным для любого элемента $a \in H_{2}(X(\mathbb{C}), \mathbb{Z})$. Следовательно, элемент $h$ четный, что противоречит выбору числа $\nu$. Итак, соотношение вида (9) сушествует. Положим

$$
h=\frac{1}{2^{\mu}}\left(l_{1} \boldsymbol{x}_{1}+\cdots+l_{s} \boldsymbol{x}_{s}\right),
$$

изменим числа $l_{1}, \ldots, l_{s}$ так, чтобы $h \in f^{*} H_{2}(Y, \mathbb{Z})$, тогда $h^{2}$ - четное число, следовательно, вьполняется сравнение

$$
l_{1}^{2} x_{1}^{2}+\cdots+l_{s}^{2} x_{s}^{2} \equiv 0\left(\bmod 2^{2 \mu+1}\right) .
$$

Так как $x_{k}^{2}=-\chi_{k} \equiv 0\left(\bmod 2^{\mu}\right), l_{k}^{2} \equiv 1(\bmod 8)$ при $k=i_{1}, \ldots, i_{n}$ и $l_{k}^{2} \equiv 0(\bmod 4)$ для других значений $k$, то из $(10)$ получаем $\chi_{i_{1}}+\cdots+\chi_{i_{n}} \equiv 0\left(\bmod 2^{\mu+2}\right)$. Теорема 2 доказана.

ДоКАЗАТЕЛЬСТво ТЕОРЕмЫ 3. Сначала введем дополнительные обозначения. Положим $\mathbb{C} / \mathbb{Z}=T$, тогда имеем $G$-эквивариантньй гомоморфизм $A(\mathbb{C})=T^{q}$, и проекция $\pi_{i}: A(\mathbb{C}) \rightarrow T$ - это проекция на $i$-й сомножитель. Множество $T^{G}$ обозначим через $F$, оно состоит из двух овалов, один из которых равен $\mathbb{R} / \mathbb{Z}$, его обозначим через $F_{0}$, а другой овал обозначим через $F_{1}$. Факторпространства $A(\mathbb{C}) / G, T / G$ обозначим через $B, S$ соответственно. Проекции $A(\mathbb{C}) \rightarrow B, T \rightarrow S$ обозначим через $f$. Благодаря этим обозначениям можем написать следующую коммутативную диаграмму, в которой 
рассматриваются когомологии с коэффициентами в $\mathbb{F}_{2}$ (они в записи диаграммы опускаются):

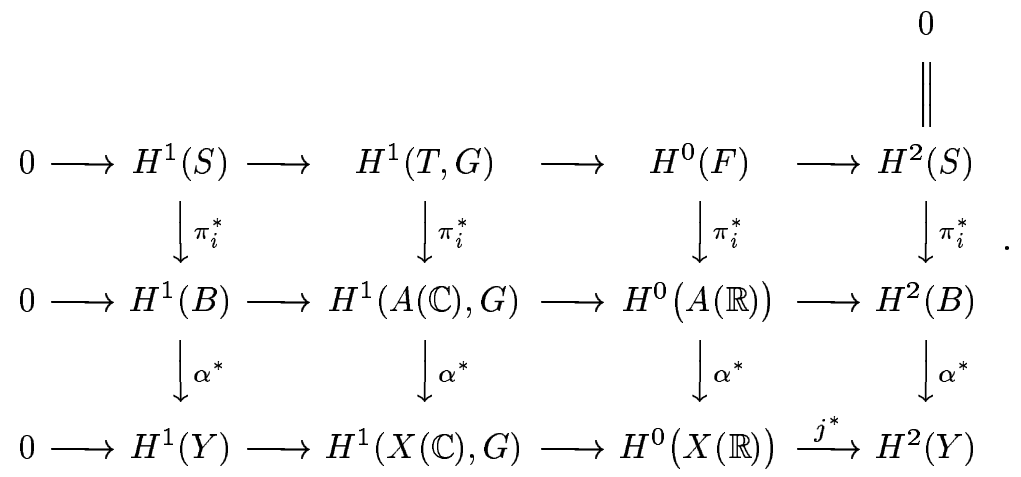

Строки в диаграмме (11) точны, они получаются из первой спектральной последовательности эквивариантных когомологий, нижняя строка - это точная последовательность (2). Пусть $\widetilde{F}_{0}$ - образующая $H^{0}(F)$, соответствующая компоненте $F_{0}$, тогда $\pi_{i}^{*}\left(\widetilde{F}_{0}\right)$ представляет собой элемент $H^{0}(A(\mathbb{R}))$, который соответствует объединению компонент $A(\mathbb{R})^{(i)}$, обозначим его через $\widetilde{A(\mathbb{R})}^{(i)}$. Элемент $\alpha^{*}(\widetilde{A(\mathbb{R})}(i)) \in H^{0}(X(\mathbb{R}))$ соответствует объединению компонент $X(\mathbb{R})^{(i)}$, обозначим его через $\widetilde{X(\mathbb{R})}^{(i)}$. Из диаграммы $(11)$ следует, что $\left.j^{*} \widehat{(X(\mathbb{R})}^{(i)}\right)=0$, т.е. класс гомологий $\left[X(\mathbb{R})^{(i)}\right]$ равен 0 в $H_{2}(Y)$. Из леммы 2 следует, что класс гомологий $\left[X(\mathbb{R})^{(i)}\right]$ равен 0 в $H_{2}(X(\mathbb{C}))$. Аналогично проверяется, что класс гомологий $[X(\mathbb{R})]$ равен 0 в $H_{2}(X(\mathbb{C}))$. Чтобы закончить доказательство теоремы 3 , нам достаточно проверить, что соотношения между классами гомологий $y_{1}, \ldots, y_{s}$, задаваемые равенствами $[X(\mathbb{R})]=0,\left[X(\mathbb{R})^{(i)}\right]=0, i=1, \ldots, q$, являются линейно независимьми. Докажем это утверждение. Так как $X-M$-поверхность, то выполняется равенство (см. [8]) $H^{1}(X(\mathbb{C}))^{G}=H^{1}(X(\mathbb{C}))$, поэтому из второй спектральной последовательности эквивариантных когомологий

$$
H^{p}\left(G, H^{q}(X(\mathbb{C}))\right) \Longrightarrow H^{p+q}(X(\mathbb{C}), G)
$$

получаем точную последовательность

$$
0 \rightarrow H^{0}(X(\mathbb{C})) \rightarrow H^{1}(X(\mathbb{C}), G) \rightarrow H^{1}(X(\mathbb{C})) \rightarrow 0
$$

Аналогичную точную последовательность имеем для группы $H^{1}(A(\mathbb{C}), G)$ :

$$
0 \rightarrow H^{0}(A(\mathbb{C})) \rightarrow H^{1}(A(\mathbb{C}), G) \rightarrow H^{1}(A(\mathbb{C})) \rightarrow 0
$$

поэтому гомоморфизм

$$
\alpha^{*}: H^{1}(A(\mathbb{C}), G) \rightarrow H^{1}(X(\mathbb{C}), G)
$$

является изоморфизмом, так как гомоморфизм

$$
\alpha^{*}: H^{1}(A(\mathbb{C})) \rightarrow H^{1}(X(\mathbb{C}))
$$


является изоморфизмом. Рассуждая аналогично с гомоморфизмами

$$
\pi_{i}^{*}: H^{1}(T, G) \rightarrow H^{1}(A(\mathbb{C}), G),
$$

мы получим, что образы $\pi_{i}^{*}\left(H^{1}(T, G)\right), i=1, \ldots, q$, порождают все вместе группу $H^{1}(A(\mathbb{C}), G)$. Тогда из диаграммы $(11)$ следует, что классы когомологий $\widetilde{X(\mathbb{R})}, \widehat{X(\mathbb{R})}(i)$ $i=1, \ldots, q$, порождают образ гомоморфизма

$$
H^{1}(X(\mathbb{C}), G) \rightarrow H^{0}(X(\mathbb{R})),
$$

т.е. ядро гомоморфизма Гизина $j^{*}$. Так как число линейно независимых соотношений между классами гомологий $y_{1}, \ldots, y_{s}$ равно $q+1$, то последнее утверждение завершает доказательство теоремы 3.

ДоКАЗАТЕЛЬСТво ТЕОРЕмЫ 4. Положим $D=C(\mathbb{C}) / G$ и рассмотрим коммутативную диаграмму

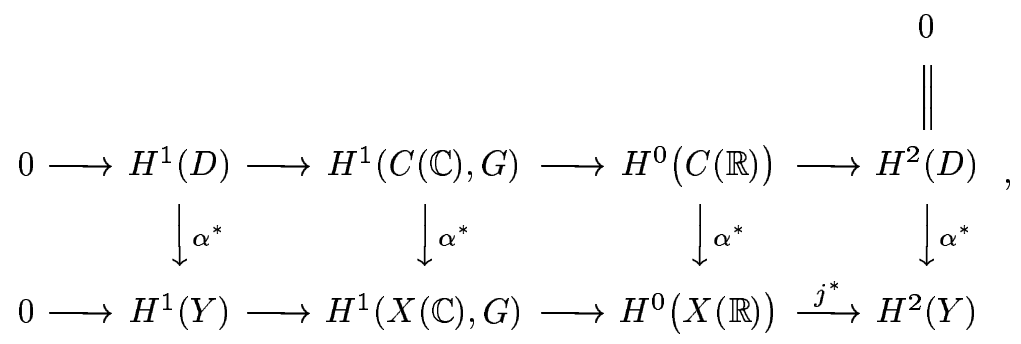

в которой строки точны и получаются из первой спектральной последовательности эквивариантных когомологий. Компоненты $C_{1}, \ldots, C_{q+1}$ определяют базис $\widetilde{C}_{1}, \ldots, \widetilde{C}_{q+1}$ группы $H^{0}(C(\mathbb{R}))$, образ $\alpha^{*}\left(\widetilde{C}_{i}\right)$ равен классу когомологий $\widehat{X(\mathbb{R})}{ }_{i}$, который получается из компонент множества $X(\mathbb{R})_{i}, i=1, \ldots, q+1$. Тогда из диаграммы (12) получаем равенства $j^{*}\left(\widetilde{X(\mathbb{R})_{i}}\right)=0, i=1, \ldots, q+1$, т.е. классы гомологий $\left[X(\mathbb{R})_{i}\right], i=1, \ldots, q+1$, равны 0 в $H_{2}(Y)$, а согласно лемме 2 они равны 0 в $H_{2}(X(\mathbb{C}))$. Остается показать, что равенства $\left[X(\mathbb{R})_{i}\right]=0$ в $H_{2}(Y)$ определяют базис соотношений между классами гомологий $y_{1}, \ldots, y_{s}$. Так как гомоморфизм

$$
\alpha^{*}: H^{1}(C(\mathbb{C})) \rightarrow H^{1}(X(\mathbb{C}))
$$

является изоморфизмом, то гомоморфизм эквивариантных когомологий

$$
\alpha^{*}: H^{1}(C(\mathbb{C}), G) \rightarrow H^{1}(X(\mathbb{C}), G)
$$

также будет изоморфизмом. Тогда из диаграммы (12) следует, что Ker $j^{*}$ порождается классами когомологий $\widehat{X(\mathbb{R})} i, i=1, \ldots, q+1$. Так как число линейно независимых соотношений между классами гомологий $y_{1}, \ldots, y_{s}$ равно $q+1$, то последнее утверждение завершает доказательство теоремы 4.

ЗАмечАниЕ. Так как $x_{1}+\cdots+x_{s}=0$, то из сравнений $\chi_{k} \equiv 0\left(\bmod 2^{\mu}\right), k=1, \ldots, s$, следует по теореме 2 сравнение $\chi(X(\mathbb{R})) \equiv 0\left(\bmod 2^{\mu+2}\right)$, но, на самом деле, выполняется сравнение $\chi(X(\mathbb{R})) \equiv 0\left(\bmod 2^{\mu+3}\right)$, что нетрудно увидеть из доказательства теоремы 2. Заметим, что это сравнение впервые было доказано в [9] с помощью сложной арифметической теории в случае, когда $H_{1}(X(\mathbb{C}), \mathbb{Z})=0$. В работе $[5]$ предложено более простое доказательство для поверхностей в свободной группе $H_{1}(X(\mathbb{C}), \mathbb{Z})$, идеи этого доказательства используются в данной работе при обосновании теоремы 2. 


\section{СПИСОК ЦИТИРОВАННОЙ ЛИТЕРАТУРЫ}

[1] Краснов В.А. О классах гомологий, определенных вещественными точками вещественного алгебраического многообразия // Изв. АН СССР. Сер. матем. 1991. Т. 55. № 2. С. 282-302.

[2] Краснов В.А. Отображение Альбанезе для вещественных алгебраических многообразий // Матем. заметки. 1982. Т. 32. № 3. С. 365-374.

[3] Краснов В.А. Отображение Альбанезе для $G M \mathbb{Z}$-многообразий // Матем. заметки. 1984. T. 35. № 5. C. 739-747.

[4] Шафаревич И. Р. (ред. ) Алгебраические поверхности. Тр. МИАН. 1965. Т. 75.

[5] Краснов В.А. О классах когомологий, определенных вещественными точками вещественной алгебраической $G M$-поверхности // Изв. РАН. Сер. матем. 1993. Т. 57. № 5. С. 210-221.

[6] Краснов В.А. Неравенства Гарнака-Тома для отображений вещественных алгебраических многообразий // Изв. АН СССР. Сер. матем. 1983. Т. 47. № 2. С. 268-297.

[7] Краснов В.А. Эквивариантные когомологии вещественной алгебраической поверхности и их приближения // Изв. РАН. Сер. матем. (в печати).

[8] Рохлин В.А. Сравнения по модулю 16 в шестнадцатой проблеме Гильберта // Функцион. анализ и его прилож. 1972. Т. 6. № 4. С. 58-64.

[9] Никулин В. В. Инволюции целочисленных квадратичных форм и их приложения к вещественной алгебраической геометрии // Изв. АН СССР. Сер. матем. 1983. Т. 47. № 1. С. 109-177.

Ярославль

Поступило

20.03 .96 